\title{
(2) \\ (อ) OPEN ACCESS \\ Cross-talk between the gut microbiota and monocyte-like macrophages mediates an inflammatory response to promote colitis-associated tumourigenesis
}

\author{
Yunben Yang ${ }_{1}^{1,2,3}$ Lili Li $^{1}{ }^{1,2,3}$ Chunjing $\mathrm{Xu}_{1}{ }^{4}$ Yunke Wang, ${ }^{1,2,3}$ Zhen Wang, ${ }^{2,3,5}$ \\ Mengyao Chen, ${ }^{1,2,3}$ Zhou Jiang, ${ }^{2}$ Jun Pan, ${ }^{2,3,5}$ Chenghui Yang, ${ }^{2,3,5}$ Xiaogian $\mathrm{Li}^{2}$ \\ Kai Song, ${ }^{6}$ Junfeng Yan, ${ }^{2,3,7}$ Wanglan $\mathrm{Xie}^{1,}{ }^{1,2,3}$ Xianguo $\mathrm{Wu}_{1}{ }^{8}$ Zhigang Chen, ${ }^{2,3,5}$ \\ Ying Yuan, ${ }^{1}$ Shu Zheng, ${ }^{3}$ Jun Yan, ${ }^{9}$ Jian Huang, ${ }^{2,3,5}$ Fuming Qiu (i) 1,2,3
}

- Additional material is published online only. To view, please visit the journal online (http://dx.doi.org/10.1136/ gutjnl-2020-320777).

For numbered affiliations see end of article.

\section{Correspondence to Professor Fuming Qiu, Department of Medical Oncology, The Second Affiliated Hospital, Zhejiang University School of Medicine, Hangzhou 310009, China; qiufuming@zju.edu.cn and Professor Jian Huang, Department of Breast Surgery and Oncology, The Second Affiliated Hospital, Zhejiang University School of Medicine, Hangzhou 310009, China; drhuangjian@zju.edu.cn \\ YY, LL and CX contributed equally.}

Received 28 January 2020 Revised 13 August 2020 Accepted 20 September 2020 Published Online First 29 October 2020

\section{Check for updates}

(C) Author(s) (or their employer(s)) 2021. Re-use permitted under CC BY-NC. No commercial re-use. See rights and permissions. Published by BMJ.

To cite: Yang Y, Li L, Xu C, et al. Gut

2021;70:1495-1506.

\section{ABSTRACT}

Objective Macrophages are among the most abundant cells in the colon tumour microenvironment, and there is a close relationship among monocytes, macrophages and the gut microbiota. Alterations in the gut microbiota are involved in tumour development, but the underlying mechanisms remain unclear. We aim to elucidate the temporal changes in macrophage subsets and functions, and how these dynamics are regulated by microbial cues in the initiation of colitis-associated cancer.

Design A mouse model of colitis-associated tumourigenesis was established to determine macrophage dynamics. The role of monocyte-like macrophage (MLM) was confirmed by targeting its chemotaxis. The effects of the gut microbiota were assessed by antibiotic treatment and faecal microbiota transplantation.

Results A selective increase in MLMs was observed in the initial stages of colitis-associated cancer, with an enhanced secretion of inflammatory cytokines. MLM accumulation was regulated by CCL2 expression of colonic epithelial cells, which was influenced by bacteria-derived lipopolysaccharide (LPS). LPS further stimulated interleukin $1 \beta$ production from MLMs, inducing interleukin-17-producing T-helper cell activation to promote inflammation. These observations were also supported by altered microbial composition associated with human colitis and colorectal cancer, evolving transcriptional signature and immune response during human colitis-associated tumourigenesis.

Conclusions The gut microbiota uses LPS as a trigger to regulate MLM accumulation in a chemokinedependent manner and generate a precancerous inflammatory milieu to facilitate tumourigenesis.

\section{INTRODUCTION}

Colorectal cancer (CRC) is one of the most commonly diagnosed malignancies and the leading cause of cancer mortality worldwide. ${ }^{1}$ A key driver of CRC development is the inflammatory response pathway. ${ }^{2}$ Arising through this type of oncogenic pathway, colitis-associated cancer (CAC) represents a comparatively ideal model in tumour biology

\section{Significance of this study}

What is already known on this subject?

- The gut microbiota is involved in intestinal inflammation and carcinogenesis.

- Monocytes are considered to be recruited to the intestine, and they differentiate into macrophages.

- Macrophages exhibit their tumour-promoting activity in advanced tumours, but information on how they are regulated in early-stage cancers exposed to microbial products is limited.

What are the new findings?

- Monocyte-like macrophage (MLM) accumulation precedes colitis-associated tumour formation and contributes to inflammatory macrophage polarisation.

- Interruption of MLM recruitment into the colon reduces colitis-associated tumour burden.

- Microbiota-induced epithelial Toll-like receptor 4 (TLR4) activation promotes MLM chemotaxis.

- TLR4 ligands derived from microbiota stimulate MLM, promoting interleukin-17-producing T-helper (Th17) cell expansion and tumour formation.

How might it impact on clinical practice in the foreseeable future?

- Understanding macrophage dynamics at early-stage colitis-associated cancer and how the gut microbiota shapes immunity via its products may have implications for future immunotherapy design.

to unravel how inflammatory responses activate tumourigenesis in the normal colonic mucosa. Chronic inflammation is known to drive low-grade tumours into high-grade intraepithelial neoplasia (HGIN) and eventually full-blown CRC. Inflammatory bowel disease (IBD), particularly ulcerative colitis (UC), has been suggested a predisposing 
factor for CRC. ${ }^{3}$ Despite advances in medical treatment, a recent population-based study showed that the expected reduction in IBD-related intestinal resection rates was not achieved. ${ }^{4}$ A considerable number of patients with IBD still require colectomy for their refractory disease or treatment for intestinal neoplasia. ${ }^{56}$ Therefore, there is an urgent need to characterise the immunological mechanisms involved in UC and inflammation-driven colon tumourigenesis.

Macrophages are a heterogeneous collection of immune cell types that are involved in the maintenance of tissue homoeostasis and bidirectional regulation of inflammation. ${ }^{78}$ Under homoeostatic conditions, newly arrived monocytes are gradually conditioned in response to signals in the local microenvironment. ${ }^{9} 10$ This results in the differentiation from monocyte-like macrophages (MLMs) into mature colonic macrophages (MCMs). ${ }^{11}$ MCMs can maintain immune homeostasis in the colonic lamina propria (LP), mainly by their hyporesponsiveness to bacterial stimulation and promotion of local regulatory T-cell proliferation. ${ }^{12}$ However, on acute intestinal inflammation, this dynamic differentiation is disturbed in sites where there is enhanced accumulation of MLMs. ${ }^{13}$ MLMs can be persistently activated by sustained exposure to microbial metabolites, inducing a cascade of inflammatory immune responses and activation of chronic inflammation. ${ }^{11} 14$ Despite these observations, a detailed characterisation of macrophage subsets in the initiation of CAC is limited, and this hinders clinical translation of targeting heterogeneous macrophages. Specifically, the roles of macrophages in colon cancer are intricate, as they are influenced by environmental signals. Information regarding these aspects is critical for designing targeted immunotherapy.

We postulated that microbe-derived factors might mediate enhanced recruitment of MLM and contribute to colon tumourigenesis, particularly in the context of inflammation. To test this hypothesis, we conducted a comprehensive analysis of the macrophage compartment from intestinal architecture disruption to tumour formation. Moreover, we also aimed to address of the following questions: (1) what is the role of macrophages in the early stage of CAC? (2) do environmental signals affect macrophage accumulation? and (3) how do bacterium derivedfactors stimulate a unique macrophage subset to promote inflammation and tumour cell proliferation? By integrating the answers to these questions, we reveal a link between microbiota-induced immune responses and inflammation-associated tumourigenesis.

\section{MATERIALS AND METHODS \\ Animal studies}

Female wild-type C57BL/6 mice were purchased from Shanghai SLAC Laboratory Animal (Shanghai, China). Newly purchased 4-week-old C57BL/6 mice were housed for 1 week in order to normalise the gut microbiota and randomised into five individuals per cage. Mice were kept in specific pathogen-free conditions with free access to a standard diet and drinking water. Mice were maintained under a 12 hour light/dark cycle at room temperature $\left(20^{\circ} \mathrm{C}-22^{\circ} \mathrm{C}\right)$. To establish azoxymethane (AOM)dextran sodium sulfate (DSS) model, mice were given an initial intraperitoneal (i.p.) injection of AOM (Sigma-Aldrich) at the dose of $12.5 \mathrm{mg} / \mathrm{kg}$ body weight. After a week, mice were given 2.5\% DSS (36 000-50 $000 \mathrm{Da}$; MP Biomedicals) in drinking water for 5 days, followed by normal drinking water for 16 days. This cycle was repeated twice.

In select experiments, mice were treated with a neutralising aCCL2 mAb (10 mg/kg/every 3 days, i.p., clone 2H5; Bioxcell) or C-C chemokine receptor type 2 (CCR2) inhibitor (propagermanium, $10 \mathrm{mg} / \mathrm{kg} / \mathrm{day}$, dissolved in drinking water; Sigma-Aldrich). ${ }^{15} 16$ In other experiments, mice were serially treated with recombinant CCL2 (rCCL2) $(10 \mu \mathrm{g} / \mathrm{kg} /$ every 3 days, i.p., catalogue 250-10; Peprotech), Toll-like receptor 4 (TLR4) specific ligand (lipopolysaccharide (LPS) -EB Ultrapure, $2 \mathrm{mg} / \mathrm{kg}$ / every 3 days, i.p.; InvivoGen), LPS ( $2 \mathrm{mg} / \mathrm{kg} /$ every 3 days, i.p.; Solarbio), TLR4 inhibitor (TAK-242, $10 \mathrm{mg} / \mathrm{kg} / \mathrm{every} 3$ days, i.p.; MedChemExpress) or cyclooxygenase-2 (COX-2) inhibitor (Celecoxib, $10 \mathrm{mg} / \mathrm{kg} /$ every 3 days, intragastric administration; Aladdin). Polyp or tumour load was determined as the sum of the size of all colon polyps/tumours presented per mouse. ${ }^{17} 18$

\section{Antibiotic treatment and faecal transfer studies}

Mice were randomly allocated to each group for antibiotic (ABX) vs vehicle $\left(\mathrm{H}_{2} \mathrm{O}\right)$ treatment, 4 weeks prior to induction with AOM-DSS. Mice in the ABX group received four-ABX cocktail in the drinking water containing metronidazole ( $1 \mathrm{~g} / \mathrm{L}$; Aladdin), ampicillin (1 g/L; Solarbio), vancomycin $(0.5 \mathrm{~g} / \mathrm{L}$; Aladdin $)$ and neomycin ( $1 \mathrm{~g} / \mathrm{L}$; Aladdin). ${ }^{17}$ To perform faecal transfer experiments, faecal samples from mice (five mice) were collected at random and resuspended in $1 \mathrm{~mL}$ of PBS (120 mg faecal sample per $\mathrm{mL}$ ). An aliquot of $200 \mu \mathrm{L}$ of faecal slurry was used for oral gavage into each mouse every other day for 2 weeks. ${ }^{19} 20$

\section{Flow cytometry}

Isolated colonic epithelial cells (CECs) and LP mononuclear cells were resuspended in FACS buffer and stained with antibodies. Flow cytometry was performed on the CytoFLEX S flow cytometer (Beckman Coulter). CytExpert software was used for data analysis. The details are provided in the online supplementary methods.

\section{RNA isolation and real-time quantitative-PCR}

Total RNA was extracted using Trizol Reagent (Invitrogen) and reverse-transcribed into cDNA using PrimeScript RT Master Mix (TaKaRa). Real-time PCR was performed using the TB Green Premix Ex Taq II system (TaKaRa) with an Applied Biosystem 7500 instrument (Applied Biosystems). ${ }^{21}$ Primer sequences were listed in online supplemental table 1 . Relative expression to $\beta$-actin was determined using the $\Delta \Delta \mathrm{Ct}$ method.

\section{Histology and immunofluorescence}

Colonic specimens were fixed with formalin, dehydrated in ethanol, embedded with paraffin and stained with H\&E. Disease diagnosis was evaluated blindly by an experienced pathologist. For immunofluorescence staining, the following primary antibodies were used: Epithelial Cell Adhesion Molecule (EpCAM) antibody (catalogue 21 050-1-AP, proteintech), MCP-1 antibody (catalogue 66 272-1-Ig, proteintech). 4', 6-diamidino-2-phenylindole, dihydrochloride (DAPI) was used as a nuclear stain. Images were collected using an orthofluorescent microscopy (Nikon), and analysed using the Image J software.

\section{Bacterial DNA sequencing}

The V3-V4 region of $16 \mathrm{~S}$ ribosomal RNA (rRNA) gene was amplified. Sequencing libraries were generated using Ion Plus Fragment Library Kit 48 rxns (Thermo Scientific) following manufacturer's recommendations. The library was sequenced on an Ion S5 XL platform. The details are described in the online supplemental methods. 


\section{Human studies}

Human gut metagenomic data collection and multiple microarray data sets integration are described in online supplemental methods.

\section{Statistical analysis}

Statistical analysis was performed with GraphPad Prism V.8 (GraphPad Software). The significant differences between groups were determined by student's t-test, one-way or two-way analysis of variance. Results were represented as mean \pm SEM, and $p<0.05$ was considered as statistically significant, $" p<0.05$, ** $\mathrm{p}<0.01, * * \mathrm{p}<0.001, * * * \mathrm{p}<0.0001$.

\section{RESULTS}

\section{Colonic MLM accumulation precedes tumour formation}

A murine model of inflammation-based tumourigenesis was induced with AOM and DSS (figure 1A). The histological analysis showed that the AOM-DSS model recapitulated progression from chronic inflammation to HGIN at different time points (online supplemental figure 1A). With disease progression, the size and number of macroscopic polyps significantly increased (figure 1A). Additionally, the expression of proinflammatory markers was considerably upregulated in the colon of mice 6 or 9 weeks after AOM-DSS induction, compared with that in healthy baseline mice (figure 1B).

As the infiltration of myeloid cells has been suggested to play a key role in carcinogenesis, we investigated myeloid cell subsets throughout disease progression to explore the immunologic mechanism underlying tumour formation. ${ }^{22}$ Cell surface markers were used as described in recent studies (online supplemental figure 1B). ${ }^{923}$ A prominent expansion of macrophages and neutrophils was observed in the stage of HGIN (figure 1C), but no significant changes were found in other subsets (eosinophils and dendritic cells). Macrophages expressed high levels of tumour necrosis factor $\alpha$ (TNF- $\alpha$ ) and prointerleukin $1 \beta$ (pro-IL1 $\beta$ ) throughout disease progression (figure 1D, online supplemental figures $1 \mathrm{CD}, 2 \mathrm{~A})$. The mRNA levels of TNF- $\alpha$ and interleukin $1 \beta$ (IL-1 $\beta$ ) in MACS-sorted colonic macrophages were significantly increased in mice with HGIN compared with that in healthy baseline mice (online supplemental figure $2 \mathrm{~B}$ ). The analysis of macrophage phenotype revealed that a reduction in anti-inflammatory CD20 $6^{\text {high }}$ macrophages was related to the temporal progression of the disease (figure $1 \mathrm{E}$, online supplemental figure 2C).

We then verified whether inflammatory macrophage infiltration could be due to alterations in macrophage subpopulations. Consistent with previous studies, we identified three subsets of macrophages based on their expression of surface markers (Population 1 (P1), MLM: $\mathrm{CD}_{4}{ }^{+} \mathrm{CD} 11 \mathrm{~b}^{+} \mathrm{F} 4 / 80^{+}$Siglec-F $\mathrm{F}^{-} 11 \mathrm{c}^{-} \mathrm{Ly} 6 \mathrm{C}^{\mathrm{hi}} \mathrm{MHCII}^{-} ; \quad \mathrm{P} 2$, $\mathrm{CD} 45^{+} \mathrm{CD} 11 \mathrm{~b}^{+} \mathrm{F} 4 / 80^{+}$Siglec-F $\mathrm{F}^{-} \mathrm{CD} 11 \mathrm{c}^{-} \mathrm{Ly} 6 \mathrm{C}^{+} \mathrm{MHCII}^{+}$; P3, MCM: $\mathrm{CD}_{4}{ }^{+} \mathrm{CD} 11 \mathrm{~b}^{+} \mathrm{F} 4 / 80^{+}$Siglec-F CD11c $\mathrm{Ly}^{-} \mathrm{C}^{-}$ $\left.\mathrm{MHCII}^{+}\right) .{ }^{9}{ }^{10}$ Although all three subtypes of macrophages were substantially increased in AOM-DSS mice by week 9, the accumulation of MLMs could be observed as early as 6 weeks after $\mathrm{AOM}$ injection (figure $1 \mathrm{~F}$, online supplemental figure $2 \mathrm{D}$ ). We then analysed the contribution of each subpopulation to inflammatory cytokine production. P1 and P2 gated cells expressed higher levels of TNF- $\alpha$ and pro-IL1 $\beta$ at the single-cell level (figure $1 \mathrm{G}$, online supplemental figure $2 \mathrm{E}, \mathrm{F}$ ). Given the absolute number of macrophage subtypes and their cytokine-producing capacity, an increase in inflammatory macrophage differentiation can largely be attributed to MLMs. The phenotypic analysis revealed MLMs as CD115 ${ }^{\text {low }} \mathrm{CD} 135^{\text {low }} \mathrm{CX} 3 \mathrm{CR} 1^{\text {int }} \mathrm{CCR} 22^{\text {high }}$ cells (online supplemental figure 3A-D). These results indicate that the enhanced recruitment of MLMs may result in a chronic inflammatory microenvironment in the initial stages of tumourigenesis.

Besides the temporal changes, we investigated the differences in proinflammatory markers, myeloid cell subsets and macrophage dynamics between tumour and normal adjacent tissues in mice 6 or 9 weeks after AOM-DSS induction (online supplemental figure 3E-J, 4A-F). Indeed, an increase in intratumoural MLMs with an enhanced secretion of inflammatory cytokines was observed in the tumour site, indicating that the tumour microenvironment favours the recruitment and maintenance of MLMs.

\section{Colonic MLM recruitment is mediated by CCL2/CCR2}

The recruitment of monocytes to the inflamed colon is controlled by chemokine-chemokine receptor interaction. CCR2 expression, but not other chemokine receptors, correlated with monocyte-differentiation stages (figure 2A, online supplemental figure 5A). Indeed, the expression of CCR2 was increased approximately 4-fold in mice with HGIN. CCL2, one major ligand for CCR2, may act as a tumour-promoting factor to drive disease progression; we therefore investigated CCL2 level at different stages (figure $2 \mathrm{~B}$ ). As the CCL2 level was increased as early as week 6, we asked whether targeting CCL2/CCR2 could alter MLM accumulation and tumour formation. As expected, in vivo treatments using anti-CCL2 antibody or CCR2 inhibitor reduced MLM infiltration, contributing to the reduction in inflammation and epithelial proliferation (figure 2C and D, online supplemental figure 5B-H).

\section{TLR4 activation leads to epithelial CCL2 upregulation and MLM recruitment}

Recent studies have demonstrated that the gut microbiota is involved in colon tumourigenesis, and modulating commensal bacteria with $\mathrm{ABX}$ leads to tumour prevention. ${ }^{24}$ Given the fundamental role of MLM in the alterations of other macrophage subsets (P2 and P3), ${ }^{10} 25$ and pivotal effect of bacteriaderived signals for monocyte-to-macrophage switch (online supplemental figure 6A), ${ }^{9}$ we postulated that microorganismderived signals might be essential for CCL2 upregulation. This might cause potent accumulation of MLMs and foster a chronic precancerous inflammatory microenvironment. The efficacy of $\mathrm{ABX}$ was verified (online supplemental figure 6B,C). Treatment with $\mathrm{ABX}$ reduced tumour load, CCL2 expression and MLM recruitment, as well as increased CD206 ${ }^{\text {high }}$ macrophages (figure 3A-D, online supplemental figure 6D). We also observed a reduction in inflammation and epithelial proliferation in AOM-DSS mice on ABX treatment (figure 3E,F).

Next, we investigated the main source of CCL2. CECs presented a considerably higher expression of CCL2, and ABX treatment did not affect CCL2 expression in non-ECs (figure 3G, online supplemental figure 6E). We further isolated CECs and found that there was almost a 10 -fold increase of CCL2 mRNA in CECs from ABX-untreated mice compared with that in CECs from $\mathrm{ABX}$-treated mice (figure $3 \mathrm{H}$, online supplemental figure $6 \mathrm{~F}$ ). The increase of CCL2 in CECs was identified by immunofluorescent staining (figure 3I). Additionally, forced CCL2 expression in ABX-treated mice increased colonic MLMs (figure 3M).

Diverse pattern recognition receptors (PRRs) are upregulated in colon tumourigenesis and their activation exacerbates tumour formation. ${ }^{26}$ To identify whether PRR activation 
A
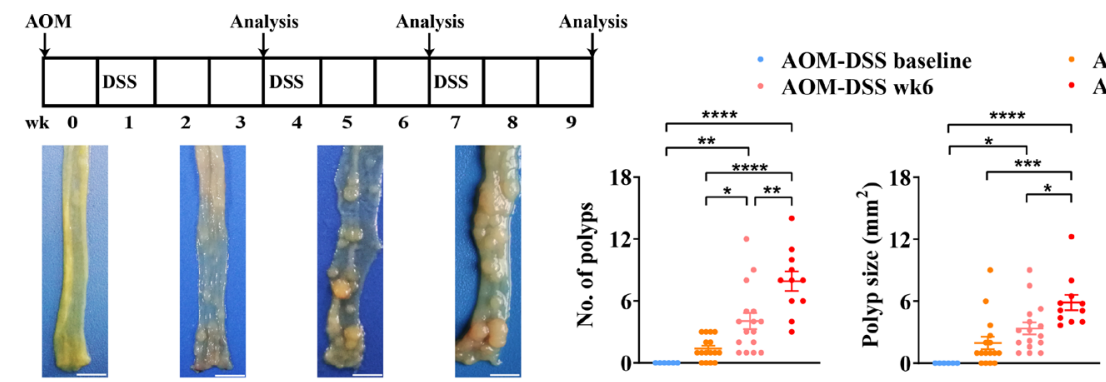

AOM-DSS wk3
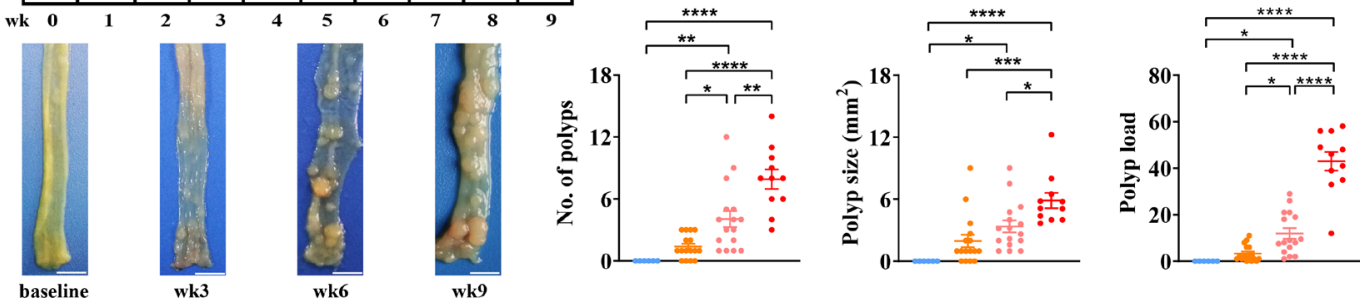

B

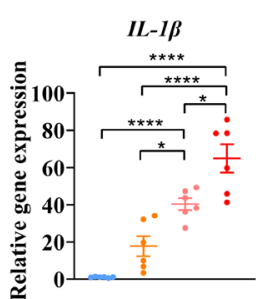

- AOM-DSS baseline
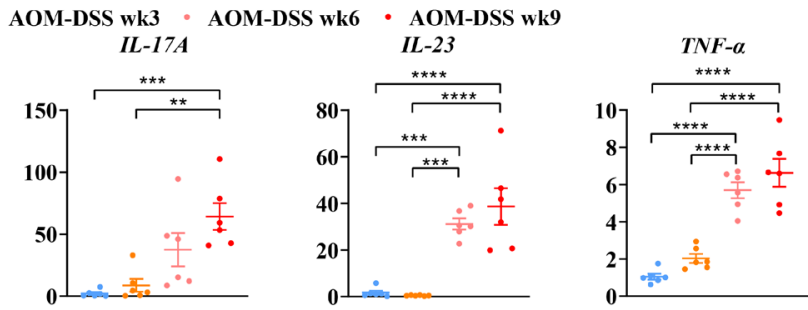

C
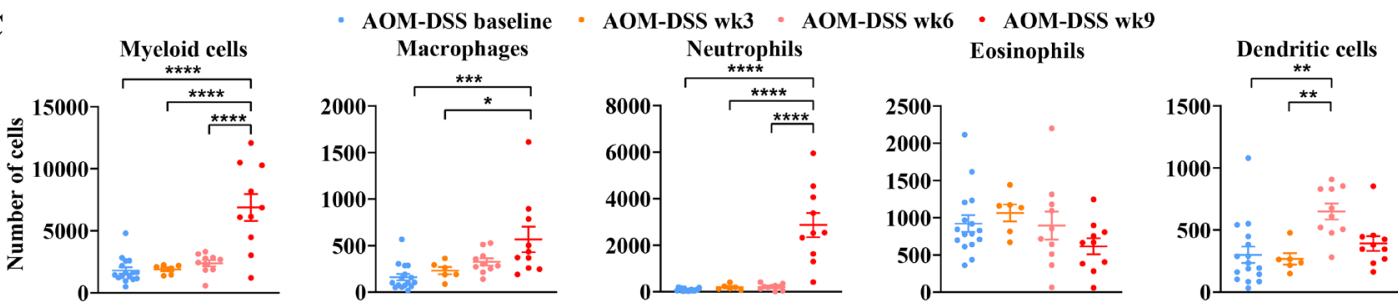

D
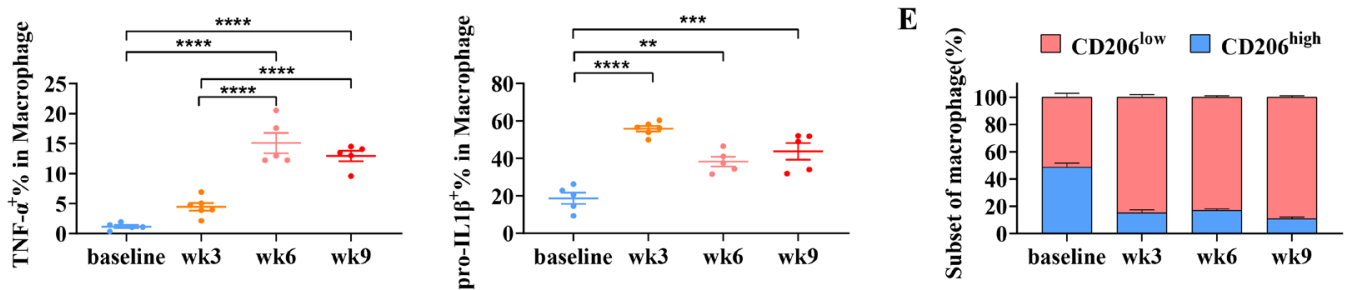

$\mathbf{F}$

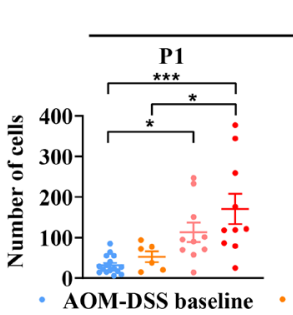

Macrophages

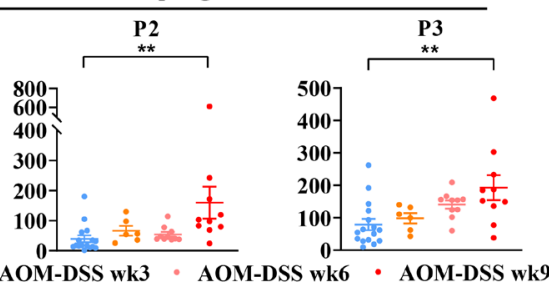

G

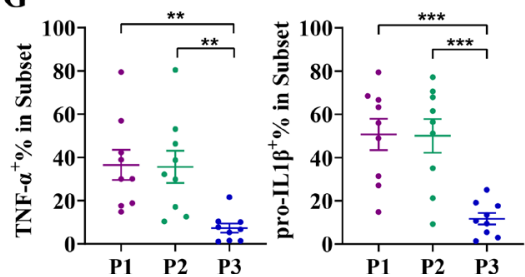

Figure 1 Early onset of monocyte-like macrophage infiltration in the colon. (A) Scheme of the AOM-DSS model. Representative images of macroscopic polyps in the AOM-DSS model throughout disease progression (scale bar, $5 \mathrm{~mm}$ ). The right panel indicates quantification of macroscopic polyps in mice (AOM-DSS baseline/wk3/wk6/wk9: $n=6 / 16 / 16 / 11)$. (B) Normalised gene expression of inflammation-related genes in the colon ( $n=6$ for each time point). Data were normalised to $\beta$-actin expression and represented as the ratio to value of baseline. (C) Absolute cell numbers of myeloid cells and distinct myeloid cell subpopulations in the colonic lamina propria (LP) as assessed by flow cytometry. A total of 100000 live colonic cells were acquired to normalise the baselines for all samples (AOM-DSS baseline/wk3/wk6/wk9: $n=16 / 6 / 10 / 10$ ). (D) Intracellular TNF- $\alpha$ and pro-IL1 $\beta$ levels of colonic macrophage throughout disease progression (AOM-DSS baseline/wk3/wk6/wk9: $n=5 / 6 / 5 / 5)$. (E) The ratio between CD206 ${ }^{\text {hi }}$ (blue) and $C D 206^{\text {low }}$ (red) subpopulations of macrophage in the colonic LP at different time points (AOM-DSS baseline/wk3/wk6/wk9: $\left.n=5 / 6 / 10 / 10\right)$. (F) Absolute cell numbers of each macrophage subset (AOM-DSS baseline/wk3/wk6/wk9: $n=16 / 6 / 10 / 10)$. (G) Intracellular TNF- $\alpha$ and pro-IL1 $\beta$ levels in macrophage subsets in mice of 9 weeks after AOM-DSS induction ( $n=9$ for each subset). Data are represented as mean $\pm S E M$, and each symbol represents one mouse; ${ }^{*} p<0.05 ;{ }^{* *} p<0.01 ;{ }^{* *} p<0.001 ;{ }^{* * * *} p<0.0001$, one-way analysis of variance (ANOVA). AOM-DSS, azoxymethane-dextran sodium sulfate; IL1 $\beta$, interleukin $1 \beta$; TNF- $\alpha$, tumour necrosis factor $\alpha$.

was involved in MLM recruitment, we first determined PRRassociated gene expression. CECs from ABX-treated mice exhibited a lower expression of PRR-related genes, most notably TLR4 and TLR7, than those from the control mice (figure 3J).
A previous study reported that the activation of epithelial TLR4 drove colon tumourigenesis ${ }^{28}$; we therefore postulated that the excessive immune reactions facilitated by the gut microbiota were due to higher epithelial TLR4 activation. Faecal extract 
A

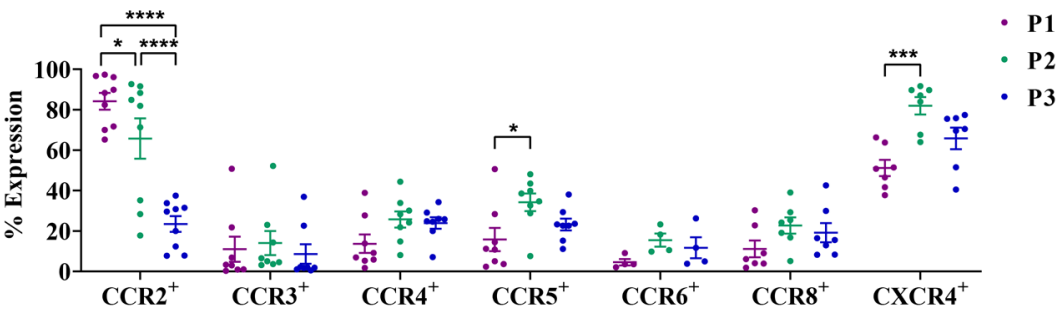

B
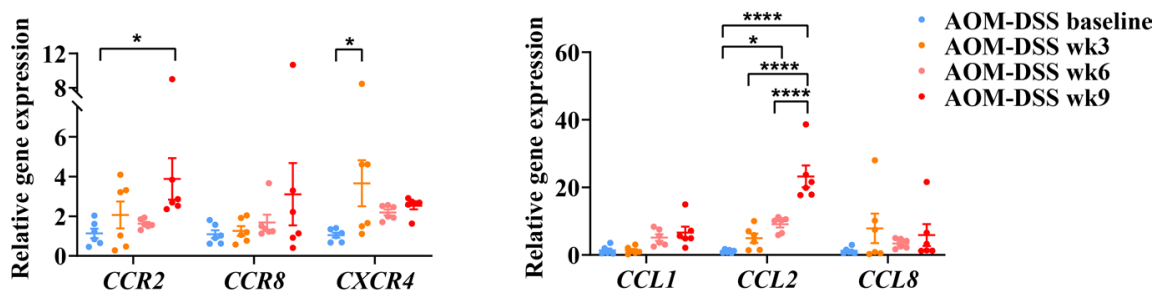

C

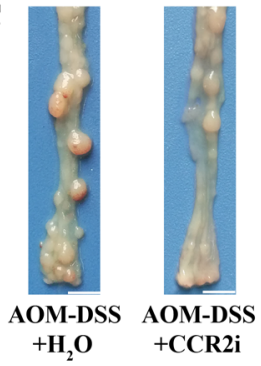

D

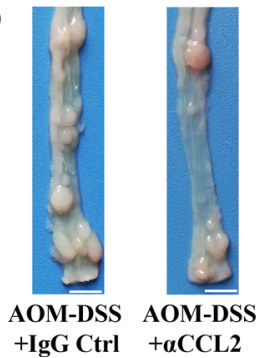

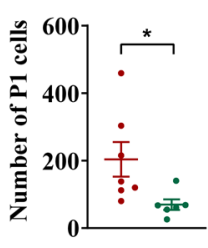
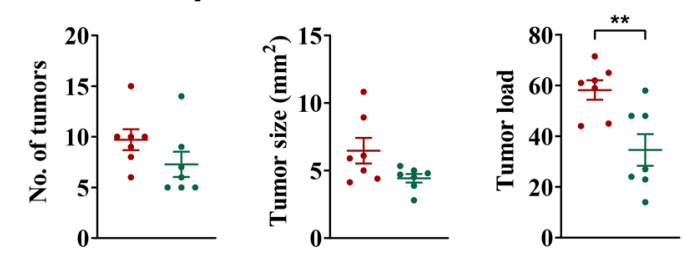

- AOM-DSS+IgG Ctrl AOM-DSS $+a C C L 2$
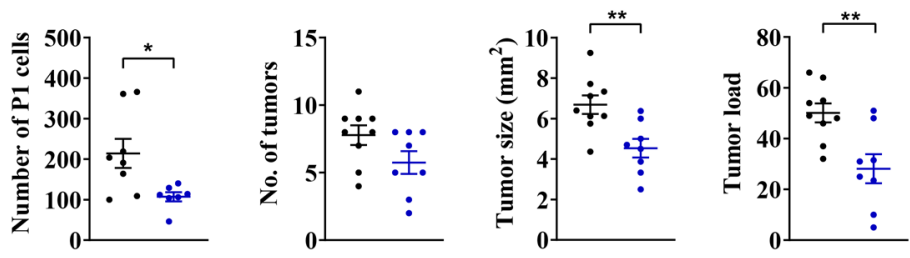

Figure 2 Colonic MLM accumulation is controlled by CCL2/CCR2. (A) Expression of chemokine receptors in each macrophage subset in mice of 9 weeks after AOM-DSS induction ( $n=4-9)$. ${ }^{*} p<0.05 ;{ }^{* *} p<0.001 ;{ }^{* * *} p<0.0001$, two-way ANOVA. (B) Normalised gene expression for chemokine and chemokine receptor in the colon throughout disease progression ( $n=6$ for each time point). Data were represented as the ratio to baseline. Twoway ANOVA. (C, D) Effect of CCR2 inhibitor (C) or $\alpha C C L 2$ (D) on MLM accumulation and colon tumourigenesis (scale bar, $5 \mathrm{~mm} ; n=6-9$ per group). ${ }^{*} \mathrm{p}<0.05 ;{ }^{* *} \mathrm{p}<0.01$, Student's $\mathrm{t}$-test. AOM-DSS, azoxymethane-dextran sodium sulfate; ANOVA, analysis of variance; CCR2, C-C chemokine receptor type 2; MLM, monocyte-like macrophage.

from tumour-bearing mice contained a high level of LPS, and its promoting effect on epithelial CCL2 could be compromised by TLR4 inhibition (figure 3K,L, online supplemental figure 6G). Next, we attempted to connect these findings to MLM accumulation and tumour growth. TLR4 ligation reversed ABX-induced MLM down-regulation and tumour inhibition. However, blocking CCL2 or CCR2 eliminated the effects of TLR4 ligation on tumour growth (figure $3 \mathrm{~N}, \mathrm{O}$, online supplemental figure $6 \mathrm{H})$. These data suggest that microbiota-derived LPS acts as a candidate for epithelial CCL2 upregulation and leads to the accumulation of MLMs in the colon.

\section{Diverse effects of stage-specific microbiota on MLM accumulation and tumourigenesis}

Although the initial microbial structure plays a key role in determining susceptibility to CAC, potential mechanisms involved in this process remain unclear. ${ }^{29}$ Therefore, we first determined the temporal changes in the microbial structure and their roles in oncogenesis. Dynamic comparisons of the microbiome in AOM-DSS mice indicated that members of the Bacteroides were increased in the later stages of disease (online supplemental figure 7A-G). At the organism-level, the enriched proportion of gramnegative microbes was found at later time points (figure 4A, online supplemental figure $7 \mathrm{H}$ ). Next, we repopulated mice gut bacteria using stools derived from different stages of tumourigenesis. Repopulation of the gut microbial flora with faeces derived from tumour-bearing mice (9 weeks after AOM-DSS induction), but not colitis-associated (3 weeks after AOM-DSS induction) or healthy baseline mice, accelerated MLM infiltration and tumour growth (figure 4B-D, online supplemental figure 8A-D).

To corroborate these findings in human disease, we used GMrepo, a database known for highly accessible and reusable human metagenomic data, to evaluate the species differences in patients with UC or CRC compared with healthy donors. ${ }^{30}$ 
A

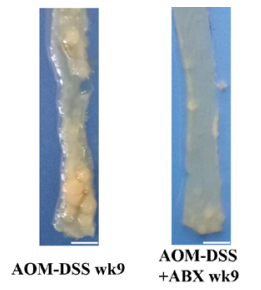

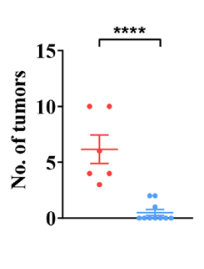
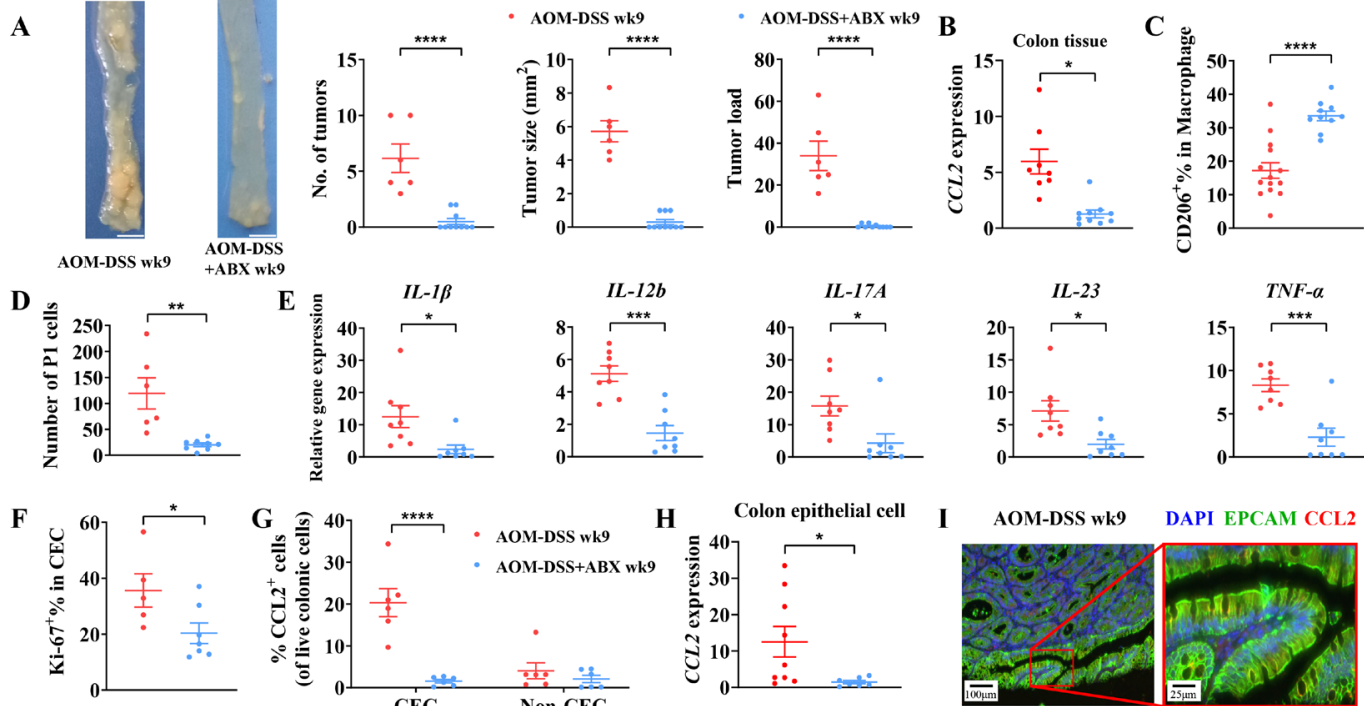

J
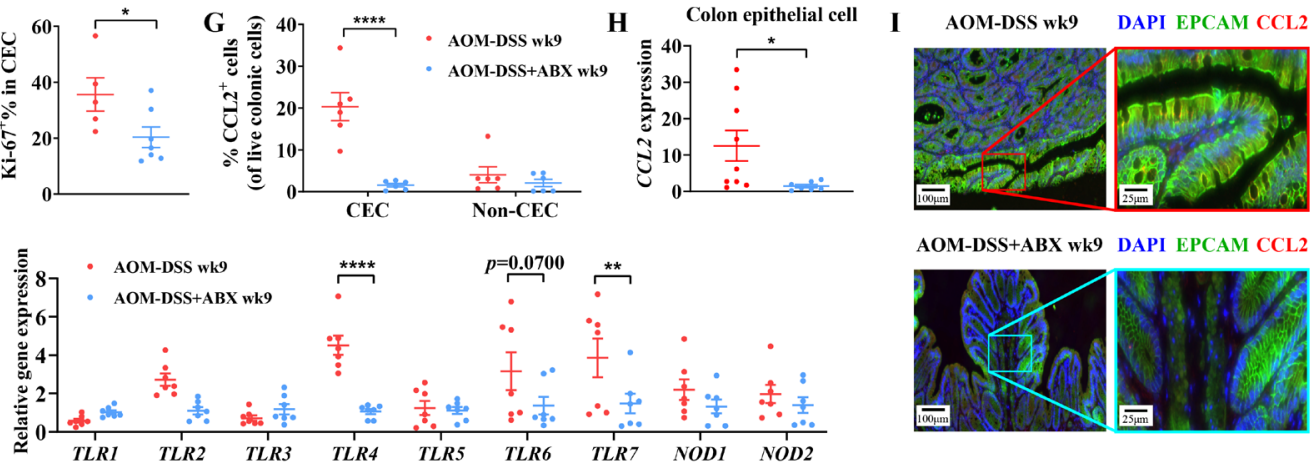

AOM-DSS+ABX wh9 DAPI EPCAM CCL2
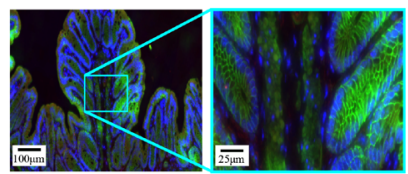

$\mathbf{K}$

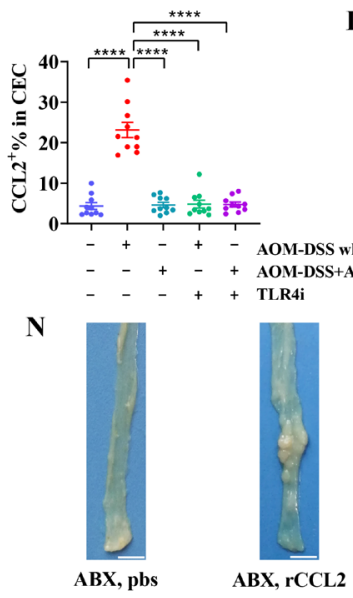

L AOM-DSS wk9

L AOM-DSS+ABX wk9 M

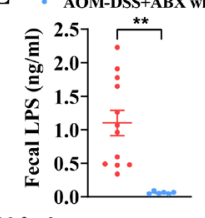

0.0

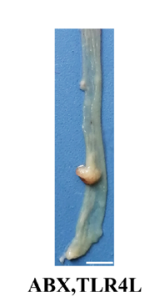

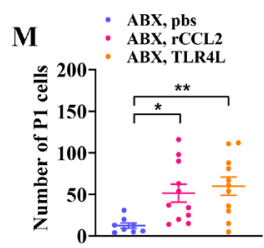

$0^{-A B X}$, pbs * ABX, rCCL2 - ABX,TLR4L

O - ABX,TLR4L+ $+\alpha C C L 2$ - ABX,TLR4L+CCR2i
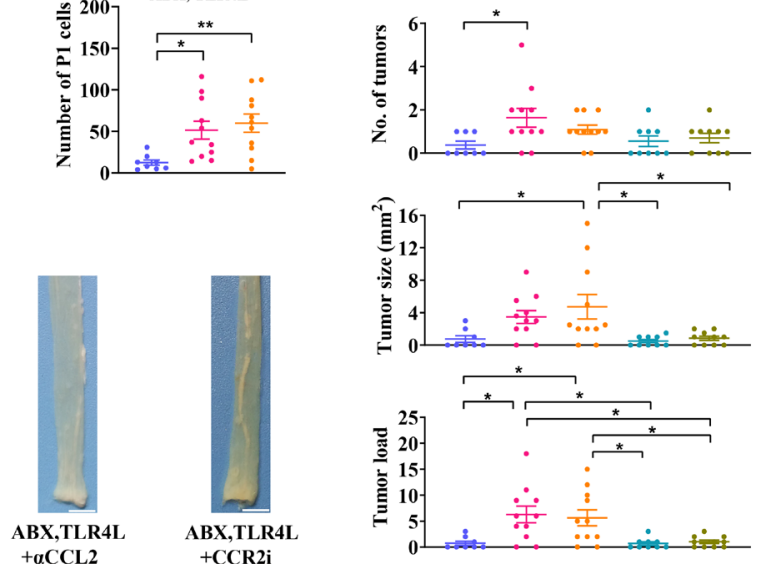

Figure 3 TLR4 activation mediates CCL2 upregulation in CECs, MLM accumulation and tumour formation. (A) Representative images of tumours in the colon of mice treated with $\mathrm{H}_{2} \mathrm{O}$ or $\mathrm{ABX}$ (scale bar, $5 \mathrm{~mm}$ ). The right panel indicates quantification of tumours in mice (AOM-DSS wk9: $\mathrm{n}=6$; AOM-DSS +ABX wk9: $n=10) .{ }^{* * *} p<0.0001$, Student's t-test. (B) CCL2 expression in colon tissues (AOM-DSS wk9: $n=8 ; A O M-D S S+A B X$ wk9: $n=10) .{ }^{*} p<0.05$, Student's t-test. (C) CD206 expression in colonic macrophages (AOM-DSS wk9: $\left.n=14 ; A O M-D S S+A B X w k 9: n=10\right) .{ }^{* * * *} p<0.0001$, Student's t-test. (D) Colonic MLMs in mice treated with $\mathrm{H}_{2} \mathrm{O}$ or $\mathrm{ABX}$ (AOM-DSS wk9: $\mathrm{n}=6$; AOM-DSS +ABX wk9: $\mathrm{n}=8$ ). ${ }^{* *} \mathrm{p}<0.01$, Student's t-test. $(E, F)$ Normalised gene expression of inflammation-related genes in colon tissues (E), and Ki-67 expression in CECs (F) from vehicle or ABX-treated AOM-DSS wk9 mice ( $n=5-8$ per group). ${ }^{*} p<0.05 ;{ }^{* *} p<0.001$, Student's t-test. (G) Intracellular CCL2 levels in different cell types as assessed by flow cytometry (AOM-DSS wk9: $n=6$; AOM-DSS +ABX wk9: $n=6)$. ${ }^{* * * *} p<0.0001$, two-way ANOVA. (H) CCL2 expression in CECs isolated from mice treated with $\mathrm{H}_{2} \mathrm{O}$ or $\mathrm{ABX}$ (AOM-DSS wk9: $n=9 ; A O M-D S S+A B X$ wk9: $n=7$ ). * $p<0.05$, Student's t-test. (I) Immunofluorescence staining of $C C L 2$ and EpCAM in colon sections from mice treated with $\mathrm{H}_{2} \mathrm{O}$ or $\mathrm{ABX}$. (J) PRR-related gene expression in $\mathrm{H}_{2} \mathrm{O}$ - or ABX-treated mice (AOM-DSS wk9: $n=7$; AOM-DSS +ABX wk9: $n=7)$. ${ }^{* *} p<0.01,{ }^{* * * *} p<0.0001$, two-way ANOVA. (K) Isolated CECs from normal mice were cultured in the presence of faecal extracts, or a combination of TLR4 inhibitor. CCL2 production was measured by flow cytometry ( $n=10$ for each group). ${ }^{* * * *} \mathrm{p}<0.0001$, one-way ANOVA. (L) Faecal LPS levels (AOM-DSS wk9: $n=12$; AOM-DSS +ABX wk9: $n=6$ ). * ${ }^{*} p<0.01$, Student's t-test. (M) ABX-pretreated AOM-DSS mice were additionally administered with rCCL2 or TLR4 ligand or vehicle. Colonic MLMs in mice (9 weeks after AOM-DSS induction) were quantified by flow cytometry ( $n=8-11$ for each group). ${ }^{*} p<0.05$, ${ }^{*} p<0.01$, one-way ANOVA. (N-0) ABX-treated AOM-DSS mice receiving rCCL2 or TLR4 ligand. In some experiments, TLR4 ligand-treated mice also received $\alpha C C L 2$ or CCR2 inhibitor. Representative images (N) and quantification (0) of tumours in different treatments (scale bar, 5 mm; $n=8-11$ for each group, 9 weeks after AOM-DSS induction). * $p<0.05$, one-way ANOVA. ABX, antibiotic; AOMDSS, azoxymethane-dextran sodium sulfate; ANOVA, analysis of variance; CCR2, C-C chemokine receptor type 2; CECs, colonic epithelial cells; IL1 $\beta$, interleukin 1 $\beta$; LPS, lipopolysaccharide; MLM, monocyte-like macrophage; TLR4, Toll-like receptor 4; TNF- $\alpha$, tumour necrosis factor $\alpha$. 


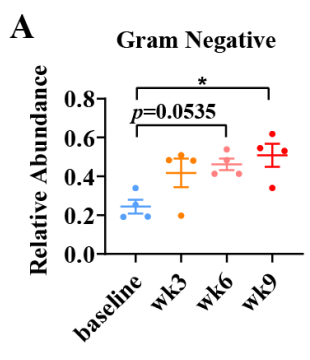

C

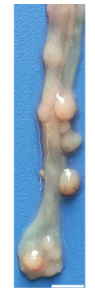

Control
B

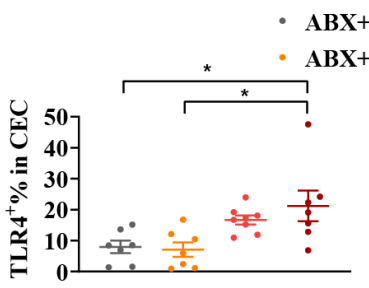

$\mathbf{A B X}+\mathbf{A} / \mathbf{D}$ baseline feces

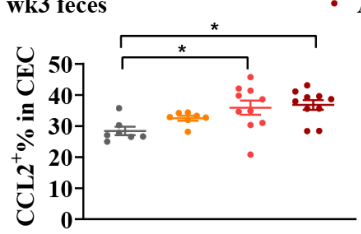

- $\mathrm{ABX}+\mathrm{A} / \mathrm{D}$ wk6 feces

- $\mathrm{ABX}+\mathbf{A} / \mathrm{D}$ wk9 feces

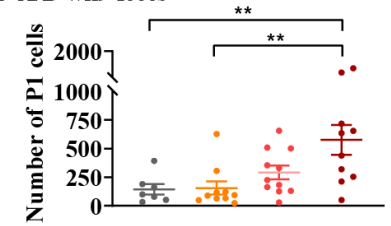

$\mathbf{E}$

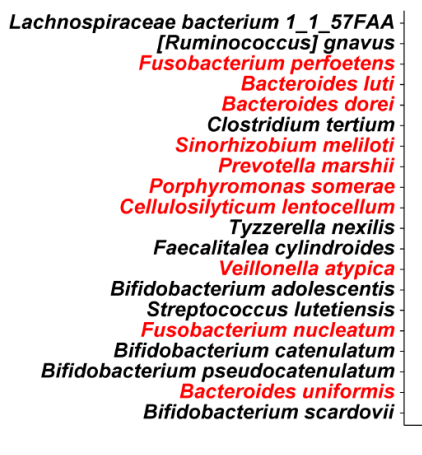

F

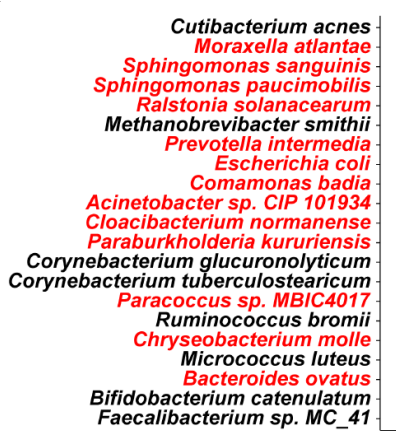

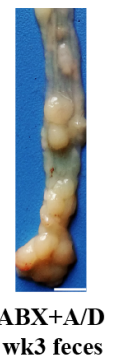

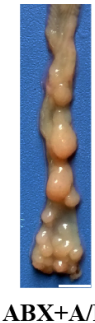

$\mathbf{A B X}+\mathbf{A} / \mathbf{I}$ wk6 feces
D Control - ABX+A/D baseline feces

- ABX+A/D wk3 feces $\quad A B X+A / D$ wk6 feces

- $\mathrm{ABX}+\mathrm{A} / \mathrm{D}$ wk9 feces
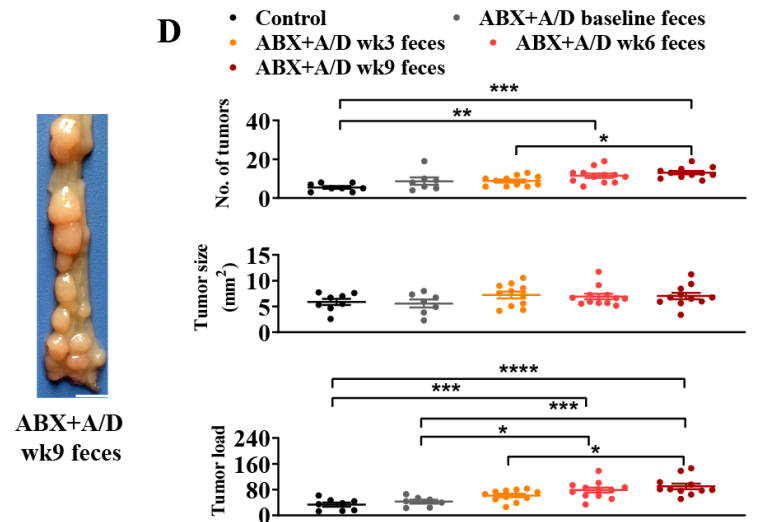

Figure 4 Diverse effects of stage-specific microbiota on MLM accumulation and tumourigenesis. (A) The proportion of gram-negative bacteria within microbiomes of stools from mice at different time points, as predicted by BugBase ( $n=4$ for each time point). (B) Mice pretreated with $A B X$ for 4 weeks were (i) repopulated with faeces from healthy baseline mice, (ii) repopulated with faeces from 3 week, 6 week or 9 week AOM-DSS mice. Mice were then induced with AOM-DSS. TLR4 and CCL2 levels in CECS, and colonic MLM levels were determined by flow cytometry ( $n=7-11$ for each group). (C, D) The ABX- or vehicle-pretreated mice were repopulated with faeces from different stages of tumourigenesis, or sham-repopulated. Mice were subsequently induced with AOM-DSS. Representative images $(C)$ and quantitative analysis (D) of tumours in different treatments (scale bar, 5 $\mathrm{mm} ; \mathrm{n}=7-12$ for each group). Data are represented as mean $\pm \mathrm{SEM} ;{ }^{*} \mathrm{p}<0.05,{ }^{* *} \mathrm{p}<0.01,{ }^{* * *} \mathrm{p}<0.001,{ }^{* * *} \mathrm{p}<0.0001$, one-way ANOVA. (E, F) Species differences in patients with ulcerative colitis (UC) (E) or colorectal cancer (CRC) (F) compared with healthy donors. Species name in red: known gramnegative bacteria. Runs indicate objects containing sequencing run data files, and each dot in plots represents one related run. 'Related runs' refer to the total number of runs that are detected positive for specific bacterial species. Multiple t-test with two-stage step-up method of Benjamini, Krieger and Yekutieli. ABX, antibiotic; AOM-DSS, azoxymethane-dextran sodium sulfate; ANOVA, analysis of variance; CECs, colonic epithelial cells; MLM, monocyte-like macrophage. 
The sequencing data revealed an increased abundance of gram-negative organisms in patients with UC, including Fusobacterium perfoetens, Bacteroides luti, Bacteroides dorei, Sinorhizobium meliloti, Prevotella marshii, Porphyromonas somerae, Cellulosilyticum lentocellum, Veillonella atypica and Fusobacterium nucleatum, whereas bacterial species enriched in healthy donors were mainly characterised by members of the Bifidobacterium (figure 4E). Similarly, bacterial species enriched in patients with CRC covered a range of gram-negative microbes, including Moraxella atlantae, Sphingomonas sanguinis, Sphingomonas pancimobilis, Ralstonia solanacearum, Prevotella intermedia, Escherichia coli, Comamonas badia, Acinetobacter sp. CIP 101934, Cloacibacterium normanense, Paraburkholderia kururiensis, Paracoccus sp. MBIC4017 and Chryseobacterium molle (figure 4F). These results may represent, at least partially, evidence in favour of the involvement of gram-negative microbes in colitis-associated tumourigenesis.

\section{TLR4 ligands derived from microbiota induce the accumulation and activation of MLMs}

Given the enrichment of LPS-producing microbes in tumourbearing mice, we hypothesised that phenotypic variance in colitis-associated tumour progression might be attributed to the level of LPS. We found that the LPS biosynthesis pathway and faecal LPS level were associated with the temporal progression of the disease (figure 5A,B). In murine CECs treated with multiple concentrations of LPS, CCL2 expression was increased at higher LPS levels (figure 5C). Similarly, the promoting effects of LPS in CCL2 regulation were validated in vivo and abrogated by TLR4 inhibition (figure 5D, online supplemental figure 9A).

The proinflammatory pathways, including COX-2 and IL-17producing T-helper (Th17), are critical for cell proliferation and colorectal neoplasia development. We sought to determine whether they were involved in the activation of MLMs by microbial stimuli. LPS induced COX- 2 and $I L-1 \beta$ expression in MLMs, which could be reversed by TLR4 inhibition (online supplemental figure 9B,C). Consequently, MACS-sorted CD4 ${ }^{+}$ cells produced more IL-17 when cultured with the supernatant from MLMs treated with LPS, and this could be reversed by IL-1 $\beta$ neutralisation (figure $5 \mathrm{E}$ ). Moreover, the in vivo inhibition of TLR4 or COX-2 signalling compromised the LPS-induced increase in pro-IL1 $\beta^{+}$MLMs, Th17 cells, Ki- $67^{+}$CECs, and tumour load (figure $5 \mathrm{~F}-\mathrm{H}$, online supplemental figure 9D-F). This suggested that TLR4/COX-2 might be a key mediator in regulating IL-1 $\beta$ and further contributing to Th17 expansion. Together, these data show the importance of microbe-derived LPS in the accumulation and activation of MLMs.

\section{Evolving transcriptional signature and immune response during human colitis-associated tumorigenesis}

The transcriptional signature showed that numerous genes were highly upregulated in both UC and UC with neoplasia (UCN) specimens compared with those in healthy controls (N) (figure 6A, online supplemental figure 10A-F). The GO analysis revealed an overrepresentation of genes involved in the immune response to microbial stimulus, and in cell migration and chemotaxis (figure 6B). The gene set enrichment analysis further revealed a notable enrichment of genes correlated with TLR and chemokine signalling pathways, Th17 cell differentiation and IL-17 signalling pathways in both UC and UCN in the leadingedge subset (figure 6C and D). Importantly, the robust increase of LPS-responsive genes in UC or UCN provided evidence that they experienced bacterial stimulation from the gut microbiota (figure 6E). Subsequently, we developed a pathway heatmap by selecting altogether 30 most typical gene sets for N-UC-UCN, indicated by distinct gene sets enriched in each group (online supplemental figure $10 \mathrm{G}, \mathrm{H})$. Finally, the relative abundance of different immune-cell types was assessed using 'Cell type Identification By Estimating Relative Subsets Of RNA Transcripts' algorithm, and an increase of inflammatory macrophages was observed in UC and UCN compared with healthy controls (figure 6F,G).

\section{DISCUSSION}

The intestinal immune microenvironment is a complicated ecology of cells that displays potential tumour-initiating capacity by orchestrating persistent inflammatory responses. ${ }^{31} 32$ A microbial programming of inflammatory processes is suggested in the colon, highlighting the complexity of microbiota-immune interactions. ${ }^{2733} 34$ Therefore, understanding the biology of tumour formation in the gut requires an exposition of the crosstalk between microbial and immune microenvironment in premalignant lesions, and possible factors that affect the functional polarisation of immune cells. In this study, we found a distinct macrophage subset, MLM, associated with inflammation-driven colon tumourigenesis. The number of MLMs was apparently increased in the earliest stages of CAC and its accumulation was dependent on CCL2 signal transduction. Blocking CCL2 signalling reduced MLM recruitment and dramatically inhibited tumour growth. Moreover, we depicted a mechanism by which dysregulated microbes activated MLMs, which in turn produce IL-1 $\beta$ to promote Th17 cell expansion. This may further aggravate dysbiosis and inflammation, resulting in a vicious cycle that contributes to tumourigenesis.

The established function of macrophage in promoting tumourigenesis occurs through direct or indirect inhibition of cytotoxic T lymphocyte responses. ${ }^{7}$ Nevertheless, our understanding of macrophages as primary immunosuppressive cells is generally derived from studies on advanced tumour, which may not adequately apply to the early stage of tumour formation. ${ }^{35}$ Studies in preclinical models and human specimens have demonstrated the diverging role of macrophages in the early stage and later stage of cancers, indicating the importance of thorough characterisation of stage-specific macrophage function. ${ }^{36} 37$ Similarly, previous studies on the role of macrophages did not distinguish between heterogeneous subsets. Here, our AOM-DSS model tended to closely reflect early-stage CAC. Therefore, we investigated the microenvironment landscape in tumour formation, with an emphasis on exploring macrophages. We observed enhanced MLM accumulation occurring before tumour formation that largely resulted in inflammatory macrophage polarisation and protumour inflammatory mediator generation, fostering an inflammatory microenvironment. Furthermore, blocking MLM recruitment considerably delayed tumour formation, highlighting the therapeutic potential of selectively targeting recruited pro-tumoural macrophage subsets, while favourably retaining resident macrophages associated with homeostasis in CAC prevention.

Chronic inflammation is a hallmark of cancer, caused by persistent inflammatory responses following exposure to microbial metabolites. ${ }^{32} 38$ In response to microbial dysbiosis, an altered immune homeostasis can trigger immunopathology such as IBD. ${ }^{39}$ Similarly, microbial product release caused by barrier disruption correlates with inflammatory myeloid cell accumulation and activation, which links to tumour-elicited inflammation. ${ }^{21}$ A key problem involved in these processes is to 
A

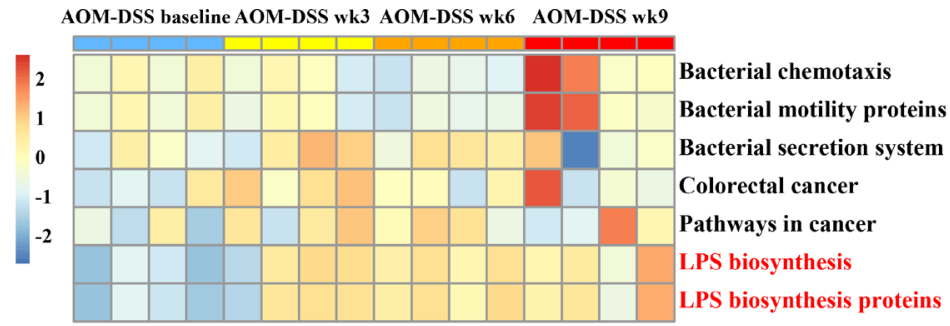

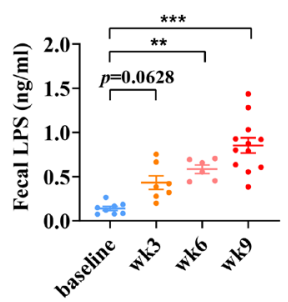

C

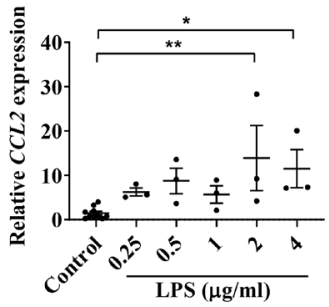

D

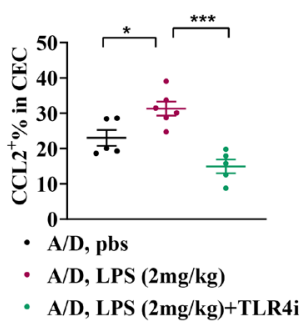

$\mathbf{E}$

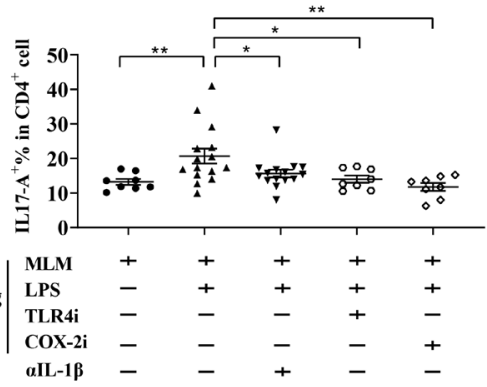

- A/D, pbs $\quad A / D$, LPS $(0.5 \mathrm{mg} / \mathrm{kg})$

- A/D, LPS $(1 \mathrm{mg} / \mathrm{kg}) \cdot \mathrm{A} / \mathrm{D}$, LPS $(2 \mathrm{mg} / \mathrm{kg})$

H

- A/D, LPS (2mg/kg)

F - A/D, LPS

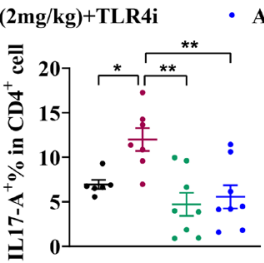

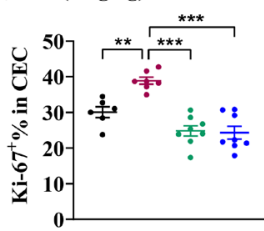

A/D, LPS (2mg/kg)+TLR4

- A/D, LPS (2mg/kg)+COX-2i

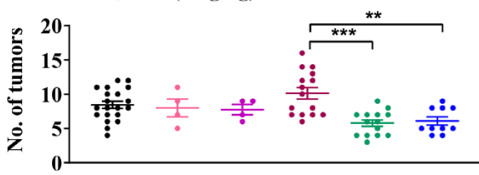

G

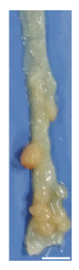

$\mathrm{A} / \mathbf{D}, \mathrm{pb}$

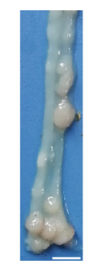

A/D, LPS $(0.5 \mathrm{mg} / \mathrm{kg})$

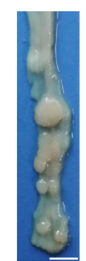

A/D, LPS $(1 \mathrm{mg} / \mathbf{k g})$

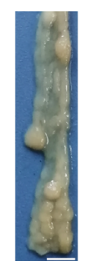

A/D, LPS $(2 \mathrm{mg} / \mathrm{kg})$

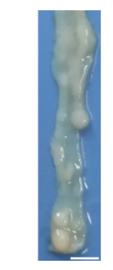

$\begin{array}{cc}\mathrm{A} / \mathrm{D}, \operatorname{LPS}(2 \mathrm{mg} / \mathrm{kg}) & \mathrm{A} / \mathrm{D}, \mathrm{LPS}(2 \mathrm{mg} / \mathrm{kg}) \\ + \text { TLR } 4 \mathrm{i} & + \text { COX-2i }\end{array}$

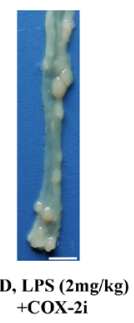

Figure 5 TLR4 ligands derived from microbiota induce the accumulation and activation of MLMs. (A) Functional profiling of microbiome throughout disease progression ( $n=4$ for each time point). (B) Faecal LPS levels (AOM-DSS baseline/wk3/wk6/wk9: $n=8 / 7 / 6 / 12$ ). (C) CCL2 expression in CECS isolated from normal mice treated with multiple concentrations of LPS for 6 hours. control: $n=10 ; n=3$ for each group of LPS treatment. (D) Intracellular CCL2 levels in CECs from AOM-DSS mice treated with LPS, or a combination of TLR4 inhibitor ( $n=5-6$ for each group, 9 weeks after AOM-DSS induction). (E) Mice were treated with $2.5 \%$ DSS for 7 days. Colonic MLMs were sorted by flow cytometry on day seven and then cultured in the presence of LPS, or a combination of TLR4/COX-2 inhibitor for 36 hours. MACS-sorted splenic CD4 ${ }^{+}$cells from normal mice were cultured with supernatant from MLM-drug cocultures under Th17 polarising conditions. As indicated, antimouse IL-1 $\beta$ was additionally added ( $n=8-15$ for each group). (F-H) AOM-DSS mice with LPS or vehicle treatment. In some experiments, LPS (2 mg/kg)-treated mice were additionally administered with a TLR4 or COX-2 inhibitor. (F) Pro-IL1 $\beta^{+}$MLMs, IL- $17 A^{+} C D 4^{+}$cells and Ki-67 ${ }^{+}$CECs in mice (9 weeks after AOM-DSS induction) were determined by flow cytometry ( $n=6-8$ for each group). Representative images $(G)$ and quantitative analysis $(H)$ of tumours (scale bar, $5 \mathrm{~mm} ; n=4-20$ for each group). Data are represented as mean $\pm S E M ;{ }^{*} p<0.05,{ }^{* *} p<0.01,{ }^{* * *} p<0.001,{ }^{* * * *} p<0.0001$, one-way ANOVA. A/D, AOM-DSS. ABX, antibiotic; AOMDSS, azoxymethane-dextran sodium sulfate; ANOVA, analysis of variance; CCR2, C-C chemokine receptor type 2; CECs, colonic epithelial cells; IL1 1 , interleukin 1 $\beta$; LPS, lipopolysaccharide; MLM, monocyte-like macrophage; Th17, interleukin-17-producing T-helper; TLR4, Toll-like receptor 4.

identify how microorganisms influence immune cell recruitment. Evidence indicate the important role of chemokines in chronic inflammation and $\mathrm{CRC}^{40} 41$; it is plausible, from inflammation to tumour formation, that the alteration in microbial composition leads to the production of chemotactic factors that recruit monocytes, which further differentiate into potentially tumourpromoting cells. Indeed, TLRs are capable of sensing microbial stimuli, thereby offering an opportunity to study the interaction between the gut microbiota and chemokine production. Studies have reported that TLRs and chemokines are closely involved in cancer development, ${ }^{27}{ }^{38}$ but with less attention on how the gut microbiota interacts with CECs and favours chemokine production. In this study, we identified CECs as a major CCL2 source and suggested that the crucial role played by the LPS/ TLR4 pathway in triggering CCL2 production ultimately leads to MLM accumulation.

The gut microbiota is involved in intestinal inflammation and carcinogenesis, by either producing carcinogenic toxins 
A
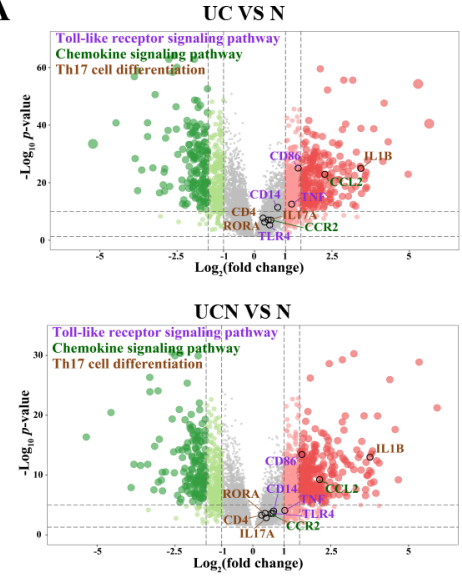

C
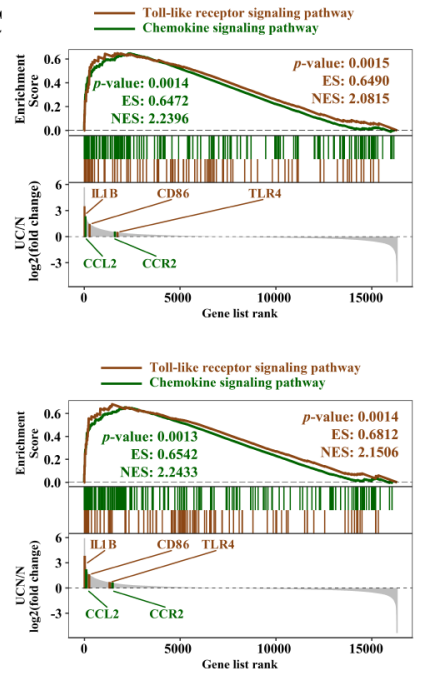

F

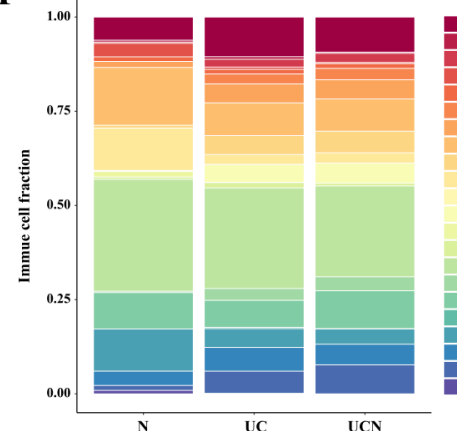

B

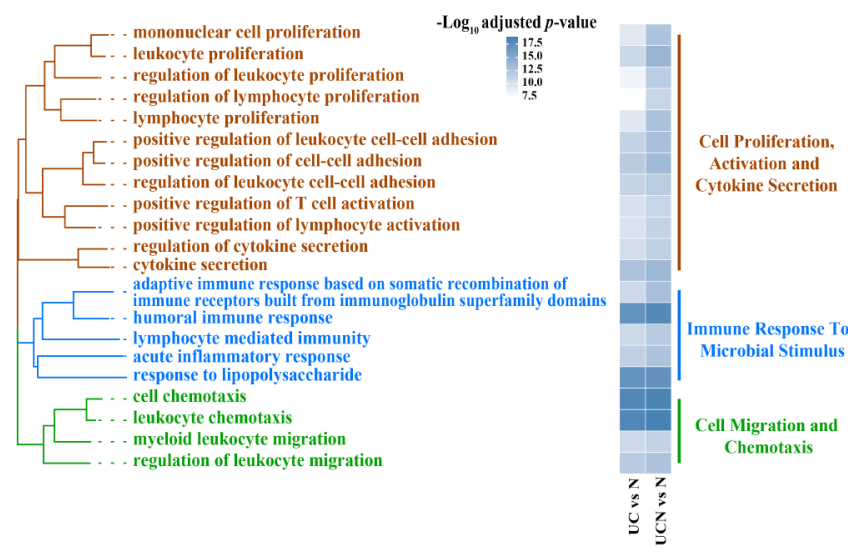

D

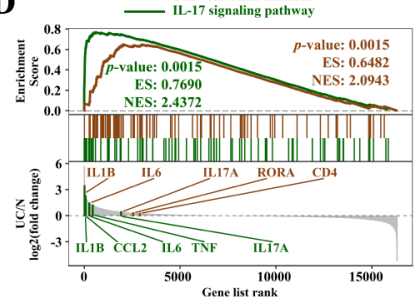

$\mathbf{E}$ 二 GSE9988_LPSVIV_VEHICLE_TREATED_NONOCYTE_UP
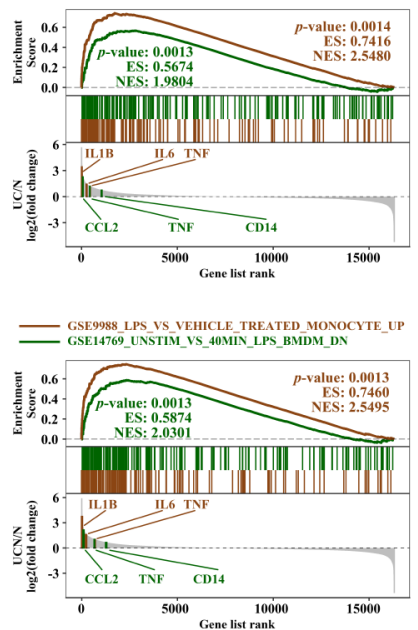

G

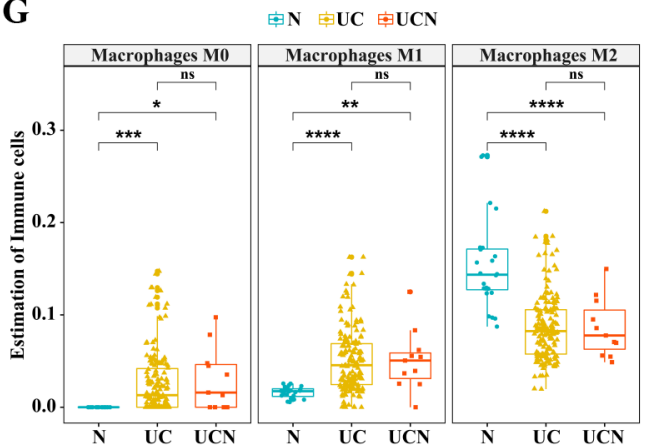

Figure 6 Evolving transcriptional signature and immune response during human colitis-associated tumourigenesis. (A) Volcano plot of differentially expressed genes (DEGs) involved in TLR and chemokine signalling pathways, as well as Th17 cell differentiation. (B) Gene Ontology (GO) enrichment analysis of DEGs between UC and normal healthy controls (N), or between ulcerative colitis with neoplasia (UCN) and N. (C-E) Gene set enrichment analysis (GSEA) plot showing the enrichment of genes upregulated in TLR and chemokine signalling pathways (C), Th17 cell differentiation and IL-17 signalling pathways (D), as well as immune responses to LPS stimulation (E). ( $F, G)$ Evolving polarisation of macrophages during colitis-associated carcinogenesis. (F) The relative abundance of immune cell from gene-expression profiles depicting the immune contexture. (G) Box plots with jittered points showing macrophage subfractions in each developmental stage (N/UC/UCN: $n=24 / 159 / 11$ ). ${ }^{*} p<0.05,{ }^{* *} p<0.01,{ }^{* * *} p<0.001,{ }^{* * * *} p<0.0001$, one-way ANOVA. ANOVA, analysis of variance; IL-17, interleukin 17; LPS, lipopolysaccharide; Th17, interleukin-17-producing T-helper; TLR, Toll-like receptor.

or modulating barrier function. ${ }^{38}{ }^{42-44}$ For example, the observation of elevated LPS in CRC specimens and the enrichment of the CRC-associated pathway related to LPS suggest its important role in CRC. ${ }^{45-47}$ In particular, the initial structure of microbiota has been shown to determine the rate of colitisassociated tumourigenesis, ${ }^{24} 2948$ indicating the protumoural effects of several bacteria and suggesting that a specific group of bacteria does have the potential to drive inflammation and promote disease progression. This may also, in turn, be affected by the accumulation of mutations during tumourigenesis, forming a dynamic network. ${ }^{49}$ Despite increasing studies have linked the gut microbiota or myeloid cells to cancer, ${ }^{2250}$ how an 
aberrant microbiota-immune crosstalk promotes inflammation and tumour cell proliferation remains unclear. We demonstrated that repopulation using faeces derived from tumour-bearing mice significantly accelerated tumour growth and that the administration of bacterial products (LPS) profoundly stimulated MLMs. In addition, we discovered the presence of microbiota-induced proinflammatory immune responses across pre-neoplastic and early stages of cancers in the colon. Combined with the therapeutic implications of targeting MLM, these findings suggest that microenvironment and suitable timing are critical factors for the treatment of CAC.

We evaluated the function of a microbiota-driven factor in the regulation of proinflammatory responses in preclinical model that may not fully reflect cancer initiation events in patients. Furthermore, we did not assess the contributions of other TLRs or bacterium-derived mediators in facilitating uncontrolled inflammation in these environments. However, in response to these limitations, recent investigations by our group and others are shedding light on the precise mechanisms of the described interplay. The combination of LPS, MLMs, and inflammatory cytokines may increase intestinal permeability, allowing the excessive release of commensal bacterial products. This promotes M2 macrophage differentiation, creating a positive feedback loop to recruit immunosuppressive cells and generate a tolerogenic environment. ${ }^{215152}$ Further studies are required to confirm our findings and elaborate possible mechanisms involved in colitis-associated tumourigenesis.

Overall, our findings establish a potential link between the gut microbiota, its products, and inappropriate immune responses in the colon (online supplemental figure 11). These results offer conceptually novel insights into the pathogenesis of CAC by uncovering the role of the gut microbiota in shaping the protumoural immune response, and they have therapeutic implications on colitis-associated tumour formation.

\author{
Author affiliations \\ 'Department of Medical Oncology, The Second Affiliated Hospital, Zhejiang University \\ School of Medicine, Hangzhou, Zhejiang, China \\ ${ }^{2}$ Key Laboratory of Tumor Microenvironment and Immune Therapy of Zhejiang \\ Province, The Second Affiliated Hospital, Zhejiang University School of Medicine, \\ Hangzhou, Zhejiang, China \\ ${ }^{3}$ Cancer Institute, Key Laboratory of Cancer Prevention and Intervention, Ministry of \\ Education, The Second Affiliated Hospital, Zhejiang University School of Medicine, \\ Hangzhou, Zhejiang, China \\ ${ }^{4}$ Department of Breast Surgery, Zhejiang Hospital, Hangzhou, Zhejiang, China \\ ${ }^{5}$ Department of Breast Surgery and Oncology, The Second Affiliated Hospital, \\ Zhejiang University School of Medicine, Hangzhou, Zhejiang, China \\ ${ }^{6}$ Department of Pathology, The Second Affiliated Hospital, Zhejiang University School \\ of Medicine, Hangzhou, Zhejiang, China \\ ${ }^{7}$ Department of Surgical Oncology, Zhuji People's Hospital of Zhejiang Province, \\ Zhuji, Zhejiang, China \\ ${ }^{8}$ Department of Clinical Laboratory, The Second Affiliated Hospital, Zhejiang \\ University School of Medicine, Hangzhou, Zhejiang, China \\ ${ }^{9}$ Department of Medicine and Department of Microbiology and Immunology, \\ James Graham Brown Cancer Center, University of Louisville, Louisville, Kentucky,
} USA

Acknowledgements We wish to thank Baohong Wang for support with sequencing analysis and technical support, Yin Gu for providing constructs.

Contributors YY, LL, CX, YW, ZW, ZJ, MC, XL, KS, JY, ZC, YY and SZ conducted the experiment and analysed the data. WX, JP, CY, XW analysed the data. JY supervised the study and edited the manuscript. JH and FQ directed the project, supervised the study. JH and FQ were responsible for the drafts of the manuscript, as well as all requested revisions.

Funding This work was supported by grants from the National Natural Science Foundation of China (81472640, 81520108024, 81672802, 81872317, 81902981, 81930079 ) and the Zhejiang Provincial Natural Science Foundation of China (No. LZ17H160004).

Competing interests None declared.

\section{Patient consent for publication Not required.}

Ethics approval All experiments were approved by the Institutional Animal Care and Use Committee of the Second Affiliated Hospital, Zhejiang University School of Medicine.

Provenance and peer review Not commissioned; externally peer reviewed.

Data availability statement Data are available on reasonable request. The 165 rRNA sequencing data were deposited to NCBI Sequence Read Archive (SRA) with accession number PRJNA597122. The list of the included human gut metagenomic runs is provided in online supplemental table 2. Data for the bioinformatics analysis using $R$ packages have been described and the complete list of analysed datasets for each group is shown in online supplemental table 3 . The data that support the findings of this study are available from the corresponding author on reasonable request.

Supplemental material This content has been supplied by the author(s). It has not been vetted by BMJ Publishing Group Limited (BMJ) and may not have been peer-reviewed. Any opinions or recommendations discussed are solely those of the author(s) and are not endorsed by BMJ. BMJ disclaims all liability and responsibility arising from any reliance placed on the content. Where the content includes any translated material, BMJ does not warrant the accuracy and reliability of the translations (including but not limited to local regulations, clinical guidelines, terminology, drug names and drug dosages), and is not responsible for any error and/or omissions arising from translation and adaptation or otherwise.

Open access This is an open access article distributed in accordance with the Creative Commons Attribution Non Commercial (CC BY-NC 4.0) license, which permits others to distribute, remix, adapt, build upon this work non-commercially, and license their derivative works on different terms, provided the original work is properly cited, appropriate credit is given, any changes made indicated, and the use is non-commercial. See: http://creativecommons.org/licenses/by-nc/4.0/.

ORCID iD

Fuming Qiu http://orcid.org/0000-0001-5793-1023

\section{REFERENCES}

1 Siegel RL, Miller KD, Goding Sauer A, et al. Colorectal cancer statistics, 2020. CA Cancer J Clin 2020;70:145-64.

2 Keum N, Giovannucci E. Global burden of colorectal cancer: emerging trends, risk factors and prevention strategies. Nat Rev Gastroenterol Hepatol 2019;16:713-32.

3 Jess T, Rungoe C, Peyrin-Biroulet L. Risk of colorectal cancer in patients with ulcerative colitis: a meta-analysis of population-based cohort studies. Clin Gastroenterol Hepatol 2012;10:639-45.

4 Murthy SK, Begum J, Benchimol El, et al. Introduction of anti-TNF therapy has not yielded expected declines in hospitalisation and intestinal resection rates in inflammatory bowel diseases: a population-based interrupted time series study. Gut 2020;69:274-82

5 Biondi A, Zoccali M, Costa S, et al. Surgical treatment of ulcerative colitis in the biologic therapy era. World J Gastroenterol 2012;18:1861-70.

6 Ungaro R, Mehandru S, Allen PB, et al. Ulcerative colitis. Lancet 2017;389:1756-70.

7 DeNardo DG, Ruffell B. Macrophages as regulators of tumour immunity and immunotherapy. Nat Rev Immunol 2019;19:369-82.

$8 \mathrm{Na}$ YR, Stakenborg M, Seok SH, et al. Macrophages in intestinal inflammation and resolution: a potential therapeutic target in IBD. Nat Rev Gastroenterol Hepatol 2019; 16:531-43.

9 Bain CC, Bravo-Blas A, Scott CL, et al. Constant replenishment from circulating monocytes maintains the macrophage pool in the intestine of adult mice. Nat Immunol 2014;15:929-37.

10 Bain CC, Scott CL, Uronen-Hansson H, et al. Resident and pro-inflammatory macrophages in the colon represent alternative context-dependent fates of the same Ly6Chi monocyte precursors. Mucosal Immunol 2013;6:498-510.

11 Isidro RA, Appleyard CB. Colonic macrophage polarization in homeostasis, inflammation, and cancer. Am J Physio/ Gastrointest Liver Physiol 2016;311:G59-73.

12 Hadis U, Wahl B, Schulz O, et al. Intestinal tolerance requires gut homing and expansion of Foxp3+ regulatory T cells in the lamina propria. Immunity 2011;34:237-46

13 Bernardo D, Marin AC, Fernández-Tomé S, et al. Human intestinal pro-inflammatory CD11chighCCR2+CX3CR1+ macrophages, but not their tolerogenic CD11C-CCR2CX3CR1 - counterparts, are expanded in inflammatory bowel disease. Mucosal Immunol 2018;11:1114-26.

14 Bain CC, Mowat AM. Macrophages in intestinal homeostasis and inflammation. Immunol Rev 2014;260:102-17.

15 Yokochi S, Hashimoto H, Ishiwata Y, et al. An anti-inflammatory drug, propagermanium, may target $\mathrm{GPI}$-anchored proteins associated with an MCP-1 receptor, CCR2. J Interferon Cytokine Res 2001;21:389-98.

16 Popivanova BK, Kostadinova FI, Furuichi K, et al. Blockade of a chemokine, $\mathrm{CCL} 2$, reduces chronic colitis-associated carcinogenesis in mice. Cancer Res 2009;69:7884-92. 
17 Bhattacharya N, Yuan R, Prestwood TR, et al. Normalizing Microbiota-Induced Retinoic Acid Deficiency Stimulates Protective CD8(+) T Cell-Mediated Immunity in Colorectal Cancer. Immunity 2016;45:641-55.

18 Cui B, Lu S, Lai L, et al. Protective function of interleukin 27 in colitis-associated cancer via suppression of inflammatory cytokines in intestinal epithelial cells. Oncoimmunology 2017;6:e1268309.

19 Wong SH, Zhao L, Zhang X, et al. Gavage of Fecal Samples From Patients With Colorectal Cancer Promotes Intestinal Carcinogenesis in Germ-Free and Conventional Mice. Gastroenterology 2017;153:1621-33.

20 Fleming C, Cai Y, Sun X, et al. Microbiota-activated CD103 ${ }^{+}$DCs stemming from microbiota adaptation specifically drive $\gamma \delta \mathrm{T} 17$ proliferation and activation. Microbiome 2017;5:46.

21 Wu P, Wu D, Ni C, et al. $\gamma \delta T 17$ cells promote the accumulation and expansion of myeloid-derived suppressor cells in human colorectal cancer. Immunity 2014:40:785-800.

22 Katoh H, Wang D, Daikoku T, et al. CXCR2-expressing myeloid-derived suppressor cells are essential to promote colitis-associated tumorigenesis. Cancer Cell 2013;24:631-44.

23 Soncin I, Sheng J, Chen Q, et al. The tumour microenvironment creates a niche for the self-renewal of tumour-promoting macrophages in colon adenoma. Nat Commun 2018;9:582.

24 Zackular JP, Baxter NT, Iverson KD, et al. The gut microbiome modulates colon tumorigenesis. mBio 2013;4:e00692-13.

25 Tamoutounour $\mathrm{S}$, Henri S, Lelouard $\mathrm{H}$, et al. Cd64 distinguishes macrophages from dendritic cells in the gut and reveals the Th1-inducing role of mesenteric lymph node macrophages during colitis. Eur J Immunol 2012;42:3150-66.

26 Fukata M, Chen A, Vamadevan AS, et al. Toll-Like receptor-4 promotes the development of colitis-associated colorectal tumors. Gastroenterology 2007;133:1869-81

27 Jobin C. Colorectal cancer: looking for answers in the microbiota. Cancer Discov 2013;3:384-7.

28 Fukata M, Shang L, Santaolalla R, et al. Constitutive activation of epithelial TLR4 augments inflammatory responses to mucosal injury and drives colitis-associated tumorigenesis. Inflamm Bowel Dis 2011;17:1464-73.

29 Baxter NT, Zackular JP, Chen GY, et al. Structure of the gut microbiome following colonization with human feces determines colonic tumor burden. Microbiome 2014;2:20.

30 Wu S, Sun C, Li Y, et al. GMrepo: a database of curated and consistently annotated human gut metagenomes. Nucleic Acids Res 2019

31 Neurath MF. Targeting immune cell circuits and trafficking in inflammatory bowel disease. Nat Immunol 2019;20:970-9.

32 Hanahan D, Weinberg RA. Hallmarks of cancer: the next generation. Cell 2011;144:646-74.

33 Tilg H, Adolph TE, Gerner RR, et al. The intestinal microbiota in colorectal cancer. Cancer Cell 2018;33:954-64.
34 Chen J, Pitmon E, Wang K. Microbiome, inflammation and colorectal cancer. Semin Immunol 2017:32:43-53.

35 Noy R, Pollard JW. Tumor-Associated macrophages: from mechanisms to therapy. Immunity 2014:41:49-61.

36 Singhal S, Stadanlick J, Annunziata MJ, et al. Human tumor-associated monocytes/ macrophages and their regulation of T cell responses in early-stage lung cancer. Sci Trans/ Med 2019;11:aat1500.

37 Franklin RA, Li MO. Ontogeny of tumor-associated macrophages and its implication in cancer regulation. Trends Cancer 2016;2:20-34.

38 Wong SH, Yu J. Gut microbiota in colorectal cancer: mechanisms of action and clinical applications. Nat Rev Gastroenterol Hepatol 2019;16:690-704.

39 Marchesi JR, Adams DH, Fava F, et al. The gut microbiota and host health: a new clinical frontier. Gut 2016;65:330-9.

40 Cremonesi E, Governa V, Garzon JFG, et al. Gut microbiota modulate T cell trafficking into human colorectal cancer. Gut 2018;67:1984-94.

41 Trivedi PJ, Adams DH. Chemokines and chemokine receptors as therapeutic targets in inflammatory bowel disease; pitfalls and promise. J Crohns Colitis 2018;12:S641-52

42 Arthur JC, Perez-Chanona E, Mühlbauer M, et al. Intestinal inflammation targets cancer-inducing activity of the microbiota. Science 2012;338:120-3.

43 Sears CL, Islam S, Saha A, et al. Association of enterotoxigenic Bacteroides fragilis infection with inflammatory diarrhea. Clin Infect Dis 2008:47:797-803.

$44 \mathrm{Xu} \mathrm{S}$, Yin W, Zhang Y, et al. Foes or friends? bacteria enriched in the tumor microenvironment of colorectal cancer. Cancers 2020;12:cancers12020372.

45 Dai Z, Coker 00, Nakatsu G, et al. Multi-Cohort analysis of colorectal cancer metagenome identified altered bacteria across populations and universal bacterial markers. Microbiome 2018;6:70

46 Zeller G, Tap J, Voigt AY, et al. Potential of fecal microbiota for early-stage detection of colorectal cancer. Mol Syst Biol 2014;10:766.

47 Kang M, Edmundson P, Araujo-Perez F, et al. Association of plasma endotoxin, inflammatory cytokines and risk of colorectal adenomas. BMC Cancer 2013;13:91.

48 Zackular JP, Baxter NT, Chen GY, et al. Manipulation of the gut microbiota reveals role in colon tumorigenesis. mSphere 2016;1:mSphere.00001-15.

49 Tjalsma H, Boleij A, Marchesi JR, et al. A bacterial driver-passenger model for colorectal cancer: beyond the usual suspects. Nat Rev Microbiol 2012;10:575-82

50 Walter J, Armet AM, Finlay BB, et al. Establishing or Exaggerating causality for the gut microbiome: lessons from human Microbiota-Associated rodents. Cell 2020;180:221-32.

51 Pushalkar S, Hundeyin M, Daley D, et al. The pancreatic cancer microbiome promotes oncogenesis by induction of innate and adaptive immune suppression. Cancer Discov 2018:8:403-16.

52 Kawano Y, Nakae J, Watanabe N, et al. Colonic pro-inflammatory macrophages

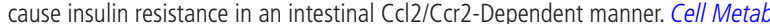
2016;24:295-310 